\section{The root of prostate cancer}

\author{
By Lauren Martz, Staff Writer
}

A lack of useful prostate cancer models has limited research and identification of new treatments for the disease. Now, researchers at the University of California, Los Angeles have identified prostatic basal cells as the originators of prostate cancer and have developed a mouse model based on these findings, which could help identify new treatments that attack the root of the disease and prevent recurrence. ${ }^{1}$

Although many prostate cancer models exist, the field lacks ones that can accurately replicate key aspects of the human disease, including initiation of the cancer and its progression to metastasis.

To address these issues, Owen Witte and colleagues at UCLA set out to identify the cells of origin for the disease. Witte is professor of microbiology, immunology and molecular genetics at UCLA and professor of molecular and medical pharmacology at the university's David Geffen School of Medicine. He is also a Howard Hughes Medical Institute investigator.

Using a method previously established by his team, ${ }^{2}$ Witte and colleagues isolated prostatic epithelial cell subtypes from healthy human tissue. The team transduced basal and luminal epithelial cells with lentiviruses expressing two oncogenes commonly upregulated during prostate cancer: protein kinase $B$ $(P K B ; A k t)$ and $E R G$. The researchers found that after injecting the lentiviruses into mice, only the basal cells developed into abnormal prostate structures resembling prostate intraepithelial neoplasia (PIN).

Transduction of the basal cells with an additional vector expressing androgen receptor (AR) caused the development of adenocarcinomas in the mice. AR is a hormone receptor that promotes prostate cell proliferation and is overexpressed in many prostate cancers.

The cancerous glands that developed from the basal cells expressed markers of human prostate cancer including $A R$, prostate-specific antigen (KLK3; PSA) and $\alpha$-methylacyl-CoA racemase (AMACR). The findings showed that transforming healthy human prostatic basal cells prior to implantation in mice can recapture the initiation and progression of human prostate cancer.

"We think that our model has an advantage because we are able to dictate and understand the history of the tumors by transforming healthy tissue with defined genes so we can test the implications of those transformations," said Andrew Goldstein, lead author on the paper and a graduate student at the Molecular Biology Institute at University of California, Los Angeles.

The data were published in Science.

The work was funded in part by the Prostate Cancer Foundation. Howard Soule, CSO of the foundation and EVP of discovery and translation, told SciBX that "Witte's team reconstructed in the lab components of prostate cancer at the cellular levels to develop glandular structures indistinguishable from the human prostate. This is a more relevant system to study the development of the disease and an incredibly important advancement in prostate cancer research."

\section{Current models}

Gail Prins, professor of physiology in the Department of Urology and the Department of Physiology and Biophysics at the University of Illinois at Chicago, told SciBX that the team has addressed one of the largest hurdles in prostate cancer research: modeling disease onset.

"This is where a lot of models are lacking," Prins said. "The most commonly used in vivo models include transgenic mice, where a gene is disrupted in or added to the prostate, and xenograft models, where you implant human cancer cells or tumor samples to grow under the skin or the kidney in the animals."

Both types of models have significant shortcomings.

"If a model is solely of animal origin, it allows you to make mechanism-of-action assessments to determine the molecular pathways involved in initiation, progression, metastasis or even death from the cancers. But it will always pertain to the animal system," Prins said. "Also, genetic manipulations to start the cancer are not ideal because they don't allow the model to follow the natural course of the disease."

Alternatively, she added, "using human specimens in xenograft models is very useful because you can study the disease in human tissues, but it is hard to figure out what started the cancer. These models give no information about the source of the disease because the tumor or cancer cells have already been established."

At the Endocrine Society meeting in San Diego in June, Prins and colleagues reported a conceptually similar model of prostate cancer to Witte's. The team isolated prostate stem cells from healthy human prostates, mixed the cells with embryonic rat prostate cancer cells and transplanted them into the kidney capsule of mice.

Without treatment, the mice developed normal human prostate tissues; when treated with testosterone and estrogen, the mice developed tumors at the site.

According to David Hung, president and CEO of Medivation Inc., the Witte report "is groundbreaking work because the main originator of the cancer might not be luminal cells, which everybody thought. It appears that the cancers arise from the basal cell subtype."

Prostate tumors are comprised primarily of the luminal cell subtype of the prostate epithelium with very little basal cell expression, which has led researchers to assume that the cancer arises from the 


\section{Box 1. Targeting the root of prostate cancer.}

With the new understanding that basal cells could be the source of prostate cancer, a University Paris Descartes group has already identified a pathway that might be involved in tumorigenesis in those cells. ${ }^{3}$

The team, led by Vincent Goffin, suggests in a paper published in the Proceedings of the National Academy of Sciences that antagonizing prolactin (PRL) could help prevent the early changes that occur in the prostate basal epithelium during cancer development by inhibiting PRLmediated activation of signal transducer and activator of transcription 5 (STAT5).

Goffin is a researcher at Institut National de la Santé et de la Recherche Médicale (INSERM) and professor at the university.

Previous studies have implicated STAT5 signaling in prostate cancer, showing that it is constitutively active in high-grade prostate cancers ${ }^{4}$ and that inhibiting its expression or activity could decrease cancer cell survival both in xenografts and in vitro. ${ }^{5}$

It also has been shown that STAT5 is the main signaling molecule that acts through the PRL receptor in prostate cancer cell culture. ${ }^{6}$

Now, the French team has shown that PRL's activity on STAT5 signaling is involved in the initiation of disease.

In transgenic mice overexpressing Prl specifically in the prostate, Stat5 was upregulated compared with that in wild-type mice, suggesting that STAT5 signaling is activated by PRL.

luminal cells and has made them a prime therapeutic target.

"It is possible that cancer cells have very different phenotypes at the different stages. While Witte has shown that cancers appear to arise in basal cells as opposed to luminal cells, as basal cells progress in their malignant phenotype, they acquire a more luminal appearance. Therefore, targeting what appears to be luminal cells may not be addressing prostate cancers in their earliest stage, and perhaps targeting basal cells may prove to be a better early intervention strategy," Hung added.

Medivation and Astellas Pharma Inc. are developing MDV3100, which is an AR antagonist that prevents androgen receptor binding to testosterone, blocks the nuclear translocation of the receptor and blocks the receptor's binding to DNA. The compound is in Phase III testing to treat castration-resistant prostate cancer (CRPC).

Samuel Denmeade, professor of oncology at The Johns Hopkins University and chief scientific advisor to Protox Therapeutics Inc., also pointed out that most available mouse models don't recapitulate human prostate cancer because the cancer progresses much more quickly in mice than in humans.

He said the model could be most valuable for identifying strategies
The transgenic mice developed prostate cancer, capturing the stages from benign prostatic intraepithelial neoplasia (PIN) to invasive carcinoma.

Also, the transgenic mice showed an accumulation of basal cells, which spread outside of the central prostate where they are normally found, suggesting that PRL might initiate tumorigenesis by altering the basal cell- and stem cell-like populations.

In double-transgenic mice expressing both prostate-specific Prl and a Prl antagonist, Stat5 activation was inhibited, the alterations in basal cell patterns and proliferation were reversed, and PIN frequency and grade were reduced. The development of prostate tumors also was completely inhibited through six months of age compared with that in the single-transgenic mice.

To move this work forward, Goffin told SciBX the next steps include deciphering the mechanism by which PRL induces amplification of the basal compartment and determining the effects of a PRL antagonist beyond six months of age.

He said an ongoing study is designed to identify longacting antagonists that could be therapeutics. In the PNAS paper, his team generated mice able to produce an antagonist because the short duration of action of available antagonists was problematic.

Goffin said the PRL antagonist has been patented and an undisclosed industrial partner has an option on an exclusive license.

$-L M$ to prevent prostate cancer (see Box 1, “Targeting the root of prostate cancer").

Protox's PORxin (PRX302), a pore-forming prodrug delivered directly to the prostate and activated by PSA, is in Phase II testing to treat localized prostate cancer and benign prostatic hyperplasia $(\mathrm{BPH})$. Kissei Pharmaceutical Co. Ltd. owns rights in Japan.

Knowing how the cancer originates, and from which cells, is important both to treat the disease and to eliminate the cells that could lead to recurrence.

"If the original source behaves differently than the majority of cells, are treatments targeting the majority going to target the source as well?" said Prins. "While one therapeutic might eliminate most tumor cells, if it doesn't kill the cells that cause the tumors, those cells could cause cancer again."

- Gail Prins,

University of Illinois at Chicago

Irwin Gelman, scientific advisor to Kinex Pharmaceuticals LLC and professor of oncology at the Roswell Park Cancer Institute, told SciBX that disease recurs in $13 \%-15 \%$ of patients after androgendeprivation therapy. "This highly aggressive form of the disease is unresponsive to all therapies," he noted.

Kinex's KX2-391, a small molecule Src inhibitor, is in Phase II trials to treat bone-metastatic CRPC. 


\section{Next steps}

Gelman and Prins both noted that no single model will be perfect.

"There is not going to be one model good enough to study every aspect of the disease," Prins told SciBX. "There is no fix-all model, nor do I think our model can answer all the questions."

According to Gelman, "it is important to understand that each of these models has inherent strengths and weaknesses. Thus, a full picture of the genetic regulation of prostate cancer progression should incorporate the advantages of each model."

Researchers told SciBX that Witte's model could be improved by adding oncogenes and finding a way to make it more reproducible.

"The one problem with this model, as with all models, is that it is still not exactly what happens in humans," Hung said. "Akt and ERG are only two genes out of many that are altered in prostate cancer. In addition, the degree of prostate gene expression could also change the outcome. This is still just a model and will not be an exact reflection of the actual disease in people, but that doesn't detract from the importance of the discovery of the cancer cells of origin."

He suggested validating the model by showing it correlates with clinical outcomes. "It is important to show that a compound effective in the model is also effective in humans with the disease. This will prove the model is useful."

In fact, the UCLA team plans to not only expand the model to test various oncogenes but also make the mice as widely available as possible.

Goldstein told SciBX the team's next step is to make the technology more robust. "We need to develop some sort of high throughput system to develop the mice that will allow us to compare the effects of therapeutics against a broad range of oncogenes," he said. "This could provide us with a good picture of what can treat particular cancers."

Goldstein said the team has not filed any patents and is not seeking patenting or licensing of the findings or model. "We want the model to be as widespread as possible," he said.

Prins has submitted a patent application covering the hormonedriven prostate cancer model and the IP is available for licensing. She said her team plans to submit the work for publication this month.

Martz, L. SciBX 3(33); doi:10.1038/scibx.2010.1000

Published online Aug. 26, 2010

\section{REFERENCES}

1. Goldstein, A. et al. Science; published online July 28, 2010; doi:10.1126/science.1189992

Contact: Owen N. Witte, University of California, Los Angeles, Calif. e-mail: owenwitte@mednet.ucla.edu

2. Lawson, D.A. et al. Proc. Natl. Acad. Sci. USA 104, 181-186 (2007)

3. Rouet, V. et al. Proc. Natl. Acad. Sci. USA; published online Aug. 9, 2010; doi:10.1073/pnas.0911651107

Contact: Vincent Goffin, University Paris Descartes, Paris, France e-mail: vincent.goffin@inserm.fr

4. Li, H. et al. Cancer Res. 64, 4774-4782 (2004)

5. Dagvadorj, A. et al. Clin. Cancer Res. 14, 1317-1324 (2008)

6. Ahonen, T.J. et al. Endocrinology 143, 228-238 (2002)

\section{COMPANIES AND INSTITUTIONS MENTIONED}

Astellas Pharma Inc. (Tokyo:4503), Tokyo, Japan Howard Hughes Medical Institute, Chevy Chase, Md. Institut National de la Santé et de la Recherche Médicale, Paris, France

The Johns Hopkins University, Baltimore, Md.

Kinex Pharmaceuticals LLC, Buffalo, N.Y.

Kissei Pharmaceutical Co. Ltd. (Tokyo:4547), Nagano, Japan

Medivation Inc. (NASDAQ:MDVN), San Francisco, Calif. Molecular Biology Institute at University of California, Los Angeles, Calif.

Prostate Cancer Foundation, Santa Monica, Calif.

Protox Therapeutics Inc. (TSX:PRX), Vancouver, British Columbia, Canada

Roswell Park Cancer Institute, Buffalo, N.Y. University Paris Descartes, Paris, France

University of California, Los Angeles, Calif. University of Illinois at Chicago, Chicago, III. 